\title{
COMMENT
}

\section{Revival of an ancient Greek art: scent detection as diagnostic tool for tuberculosis}

\author{
Daniel J. C. Berkhout ${ }^{1,2}$, Marc A. Benninga ${ }^{1}$, Nanne K. H. de Boer ${ }^{3}$ and Tim G. de Meij ${ }^{2}$ \\ Pediatric Research (2018) 84:4-5; https://doi.org/10.1038/s41390-018-0015-y
}

In 2015, the World Health Organization (WHO) included a health target in the newly adopted Sustainable Development Goals, striving to end the tuberculosis (TB) epidemic by the year 2030. In low-income countries, TB continues to be one of the most prevalent infectious diseases with leading mortality rates in the pediatric population. A key reason for these unacceptable high morbidity and mortality rates is the poor sensitivity of available diagnostic tests to detect TB. In these countries, pediatric TB is primarily diagnosed based on clinical signs and smear microscopy, consequently resulting in a large proportion of undiagnosed patients. This illustrates the need for new strategies towards tuberculosis control, including development of new diagnostic modalities. In the interesting article of Mgode et al., ${ }^{1}$ the potential of olfactory sense of trained African giant pouched rats (Cricetomys ansorgei) was evaluated as a complementary method in the diagnostic workup of pediatric TB. ${ }^{1}$ They showed that implementation of trained rats could increase the detection rate of pediatric TB by $68 \%$, compared to a single-test protocol using smear microscopy. Given the severe health implications of TB, such an increase in detection rate is of considerable clinical importance. Interestingly, already around the year $400 \mathrm{BC}$, the ancient Greek Hippocrates described the potential of the scent of sputum as diagnostic tool in TB.

Over the past years, an increasing number of studies demonstrated the potential of scent molecules, or more specifically volatile organic compounds (VOCs), as a diagnostic biomarker for a wide variety of clinical conditions, including metabolic, infectious, and inflammatory diseases and malignancies. VOCs are carbon-based chemicals, volatile at ambient temperatures, and source of the majority of surrounding odors. Being produced during metabolic processes and excreted through all conceivable bodily excrements (e.g., sputum, feces, sweat, urine), they may serve as an ideal clinical biomarker for several pathophysiological processes. VOCs derived from a particular bodily substrate may either have an endogenous (local or systemic) or exogenous origin. In order to discriminate diseased from healthy state, VOCs derived from different substrates usually provide different outcomes. For example, in intestinal diseases such as inflammatory bowel disease, the discriminative accuracy of fecal VOCs is superior over that of exhaled breath and urinary volatiles.

In the study by Mgode et al., ${ }^{1}$ sputum was chosen as a substrate of interest, which contains VOCs from both endogenous and exogenous sources. Endogenous VOCs in the sputum include volatiles originating from local pulmonary (patho)physiological processes, whereas systemic VOCs are produced during metabolic processes elsewhere in the body before being transported by the bloodstream towards the alveoli and eventually the sputum. Examples of exogenous sources include resident pulmonary microbes, inhaled VOCs, and medicinal metabolites.

In TB patients, sputum presumably contains disease-specific exogenous VOCs derived from the TB-causing pathogens residing in the lungs. Mycobacterium tuberculosis, the most commonly isolated causative agent, is transmitted through aerosol droplets into the alveoli where they enter and proliferate in alveolar innate immune cells. Subsequently, infected cells may form a typical nodular granulomatous structure. In case of uncontrolled growth, this may eventually result in lymphatic and blood vessel invasion, allowing for disease dissemination. Therefore, in addition to exogenous microbial VOCs, the sputum of TB patients may also contain local VOCs derived from pulmonary pathophysiological processes and systemic VOCs derived from other infected tissues.

Presumably, presence of TB-specific VOCs with both endogenous and exogenous origins allowed for the differentiation between TB and non-TB cases by giant pouched rats. However, a relatively high false-positive detection rate of approximately $25 \%$ by rats was demonstrated in previous studies. ${ }^{2}$ Other mycobacterial spp. and non-mycobacterial spp. presumably produce similar VOC profiles, or may provoke comparable hostspecific metabolic reactions, consequently impeding accurate identification of $M$. tuberculosis in sputum samples. The essential role of both host-specific and pathogen specific VOCs in the identification of TB cases by $C$. ansorgei is well illustrated in a study in which trained rats were presented TB-negative sputum samples spiked with $M$. tuberculosis culture isolates and natural TB sputum samples. Rats were able to discriminate the latter samples from controls with higher accuracy than the prepared samples, indicating that the inflammatory host response contributes a TBspecific VOC profile. Several key sputum volatiles, differentiating TB cases from non-TB cases by trained rats, were identified using gas chromatography-mass spectrometry (GC-MS). After isolation of these VOCs they were presented to trained rats. Interestingly, only a particular blend of TB-specific volatiles, provided in measured concentrations, allowed for an adequate reaction from these rats. ${ }^{3}$ This illustrates the challenge to unravel and identify the volatiles of interest, evoking the desired detection response in rats.

Multiple other studies have attempted to identify TB-specific VOCs using chemical analytical techniques such as GC-MS in a

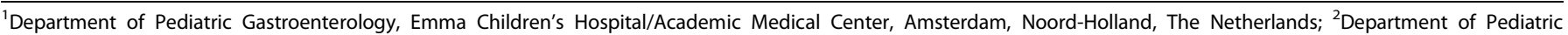

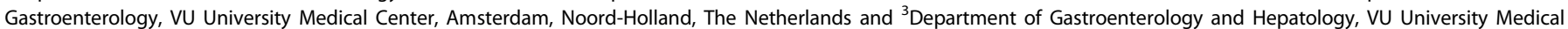
Center, Amsterdam, Noord-Holland, The Netherlands

Correspondence: Daniel J. C. Berkhout (d.berkhout@vumc.nl)

Received: 5 March 2018 Accepted: 22 March 2018

Published online: 23 May 2018 
variety of bodily substrates. Although chemical analytical devices allow for the identification of individual VOCs based on their physiochemical properties, important limitations include their high purchase costs, expensive and time-consuming measurements, complex (statistical) analysis, and the intricate outcome interpretation. The electronic nose (eNose) device may be an interesting alternative for chemical analytical techniques, especially in low-income countries such as Tanzania. In contrast to chemical analytical techniques, an eNose device contains an array of VOC sensors allowing for the identification of gaseous mixtures as a whole based on pattern recognition algorithms. They allow for bedside and high-throughput analysis and are relatively inexpensive to purchase and implement in clinical practice. However, the main limitation of eNose devices is their inability to identify individual VOCs, restraining the capability to assess which volatiles are discriminative between both subgroups. Identification of such discriminating VOCs would allow for the development of a tailored eNose, enabling quick and accurate detection of TB. Therefore, an essential next step is the identification of discriminative key volatiles identified using chemical analytical techniques.

Previously, 13 VOCs obtained from the sputum were identified as unique for TB patients; hypothetically, using these discriminative VOCs, eNose sensors allowing for accurate and real-time detection of TB could be developed. ${ }^{3}$ Moreover, whereas rats previously demonstrated an inadequate and inaccurate response after exposure to these key volatiles individually, an eNose can be trained to provide a signal if a specific VOC concentration threshold is exceeded. This would hypothetically result in an increased discriminative accuracy compared to the olfactory sense of $C$. ansorgei. An important advantage of eNose devices over sniffing animals is their consistent, objective, and reproducible results. In contrast to an eNose, detection animals may only have a limited lifespan. Consequently, a continuous supply chain needs to be set up, guaranteeing the production and training of standardized animals with identical detection rates. ${ }^{4}$ Other limitations complicating the implementation of animals in daily clinical practice include behavioral and mood variation between animals, attention span limitations resulting in relatively lowthroughput analysis, and the need for an individual performance assessment (calibration) per animal, hampering mass production. ${ }^{4}$ Another non-negligible limitation relevant to sniffing animals is the difficulty for medical societies to accept unconventional methods to be implemented in daily clinical practice, or even replace widely accepted methods at some point. Since majority of these limitations are not applicable to eNose devices, we consider the eNose to harbor great potential as future diagnostic TB tool.

However, implementation of an eNose device in the diagnostic process of TB is not an entirely new concept. So far, a few studies addressed the diagnostic potential of such devices in TB. In one particular study, an eNose containing 12 metal-oxide sensors allowed for the detection of TB cases with corresponding sensitivity and specificity values of $76.5 \%$ and $87.2 \%$, respectively. ${ }^{5}$ Interestingly, whereas only adult subjects were included in that study, a merely pediatric population was studied by Mgode et al. ${ }^{1}$ In general, diagnosing pediatric TB is more challenging since children have more difficulty in producing a sputum volume sufficient enough for smear microscopy and samples from pediatric TB cases are more often characterized by their paucibacillary. Therefore, future eNose studies should focus on TB detection in the pediatric population.

To conclude, the potential of trained giant pouched rats in enhancing the detection of pediatric TB is well illustrated by Mgode et al. ${ }^{1}$ Nonetheless, the use of sniffing animals in daily practice also entails challenges and hurdles needed to be overcome prior to clinical application. Future studies should therefore also focus on eNose devices, potentially allowing for fast and reproducible detection of TB in the pediatric population.

\section{ADDITIONAL INFORMATION}

Competing interests: The authors declare no competing interests.

Publisher's note: Springer Nature remains neutral with regard to jurisdictional claims in published maps and institutional affiliations.

\section{REFERENCES}

1. Mgode, G. F., Cox, C. L., Mwimanzi, S. \& Mulder, C. C. Pediatric tuberculosis detection using trained African giant pouched rats. Pediatr. Res. (2018). https://doi. org/10.1038/pr.2018.40 [e-pub ahead of print].

2. Poling, A. et al. Using giant African pouched rats to detect human tuberculosis: a review. Pan Afr. Med. J. 21, 333 (2015).

3. Mgode, G. F. et al. Mycobacterium tuberculosis volatiles for diagnosis of tuberculosis by Cricetomys rats. Tuberculosis (Edinb.) 92, 535-542 (2012).

4. Oh, Y., Lee, Y., Heath, J. \& Kim, M. Applications of animal biosensors: a review. IEEE Sens. J. 15, 637-645 (2015).

5. Bruins, M. et al. Diagnosis of active tuberculosis by e-nose analysis of exhaled air Tuberculosis (Edinb.) 93, 232-238 (2013). 